\title{
SGLT-2 inhibitors in diabetes: a focus on renoprotection
}

\section{SUMMARY}

Type 2 diabetes mellitus is an important public health problem, with a significant impact on cardiovascular morbidity and mortality and an important risk factor for chronic kidney disease. Various hypoglycemic therapies have proved to be beneficial to clinical outcomes, while others have failed to provide an improvement in cardiovascular and renal failure, only reducing blood glucose levels.

Recently, sodium-glucose cotransporter-2 (SGLT2) inhibitors, represented by the empagliflozin, dapagliflozin, and canagliflozin, have been showing satisfactory and strong results in several clinical trials, especially regarding the reduction of cardiovascular mortality, reduction of hospitalization due to heart failure, reduction of albuminuria, and long-term maintenance of the glomerular filtration rate. The benefit from SGLT2 inhibitors stems from its main mechanism of action, which occurs in the proximal tubule of the nephron, causing glycosuria, and a consequent increase in natriuresis. This leads to increased sodium intake by the juxtaglomerular apparatus, activating the tubule glomerular-feedback and, finally, reducing intraglomerular hypertension, a frequent physiopathological condition in kidney disease caused by diabetes. In addition, this class of medication presents an appropriate safety profile, and its most frequently reported complication is an increase in the incidence of genital infections.

Thus, these hypoglycemic agents gained space in practical recommendations for the management of type 2 diabetes mellitus and should be part of the initial therapeutic approach to provide, in addition to glycemic control, cardiovascular outcomes, and the renoprotection in the long term.

\section{INTRODUCTION}

Type 2 diabetes mellitus is considered an important public health problem that is aggravated by a context of a populational aging, sedentary lifestyle, and accumulation of body fat. In 2014, the estimated prevalence of the disease was 422 million, $8.5 \%$ of the world population, which is twice the number from the beginning of the 1980s. In addition, 1.5 million deaths recorded in 2012 were attributed to diabetes, $43 \%$ of which were individuals aged less than 70 years. In the long term, the disease is an important risk factor for retinopathy, cardio-cerebrovascular disease, and kidney disease' ${ }^{1}$.

Chronic kidney disease (CKD) has worldwide prevalence estimated at $10 \%$, with a predilection for individuals over 60 years old ${ }^{2}$, a condition with complex and multifactorial physiopathology, in addition to a systemic impact in several organs and apparatus. Hospitalization, risk of death, and cardiovascu- 
lar events are increased in CKD, especially with the progression of the disease to stages of glomerular filtration rate lower than $45 \mathrm{ml} / \mathrm{min}$ per $1.73 \mathrm{~m}^{2}$.

Diabetes mellitus has a relevant impact on cardiovascular morbidity and mortality, especially when associated with renal disease ${ }^{4}$. In Brasil, the magnitude of the problem is visible in the statistics of endstage kidney disease: $30 \%$ of patients on a permanent dialysis program have diabetes as the etiology, which is the second main cause for end-stage kidney disease requiring renal replacement therapy ${ }^{5}$.

The treatment of this metabolic disorder is represented by an extensive list of drugs of different pharmacodynamics, whose main goal is to optimize glycemic control. Sodium-glucose cotransporter-2 (SGLT2) inhibitors, a class of oral hypoglycemic agents that have recently become popular, have an insulin-independent effect. Their main mechanism of action is to inhibit SGLT2 in the proximal portion of the nephron, with consequent glycosuria and natriuretic effect and attenuation of glomerular hyperfiltration, improvement of blood pressure levels, and weight reduction. Thus, this class of medication has been a surprise in several trials due to their broad renal and cardiovascular benefits ${ }^{6}$.

Next, we review this class of medication, bringing the major clinical outcomes described in the literature, with a focus on renoprotection.

\section{THE KIDNEY AND THE GLUCOSE METABOLISM}

Approximately $80 \%$ of endogenous glucose is of hepatic origin, while $20 \%$ is renal. In the kidney, the production occurs mainly in cortical cells by adren- ergic stimulation, while the glucose consumption occurs significantly in the renal medulla. With a normal glomerular filtration rate (180 liters per day) and glycemia around $100 \mathrm{mg} / \mathrm{dL}, 180 \mathrm{~g}$ of glucose is filtered daily, with total reabsorption in the proximal tubule level? ${ }^{7}$. This balance occurs by the SGLT2 action in the S1 segment of the proximal tubule, with $80 \%$ to $90 \%$ participation in the glucose reabsorption, while SGLT1 has minimum participation of $10-20 \%$ in the segments S2 and S3. The function of SGLT 1 and 2 depends on the $\mathrm{Na}^{+}-\mathrm{K}^{+}$-ATPase pump of the basolateral membrane: the sodium $(\mathrm{Na})$ output from the pump reduces intracellular $\mathrm{Na}$, causing the sodium from the tubular to enter the cell with the glucose; this finally reaches the interstitium at the basolateral membrane through the glucose transporter (GLUT) ${ }^{8}$. A glucose value of $180 \mathrm{mg} / \mathrm{dL}$ is considered to be the limit for renal reabsorption capacity; anything above that will cause glycosuria9 .

\section{RENAL INVOLVEMENT IN DIABETES MELLITUS}

Kidney disease caused by diabetes is characterized by structural and functional alterations, whose manifestations include albuminuria and decreased glomerular filtration rate ${ }^{10}$. The initial pathophysiological events stages that lead to CKD progression are divided into early (hemodynamic and metabolic) and late (cell and tissue remodeling) ${ }^{11}$.

In a scenario of hyperglycemia, tubular glucose reabsortive capacity (Tmg) increases with greater glucose reabsorption and, therefore, sodium. Thus, a lower concentration of sodium chloride reaches the macula densa and, through tubule-glomerular feed-

FIGURE 1. NORMAL GLOMERULAR HEMODYNAMICS (1A) AND KIDNEY DISEASE CAUSED BY DIABETES (1B).
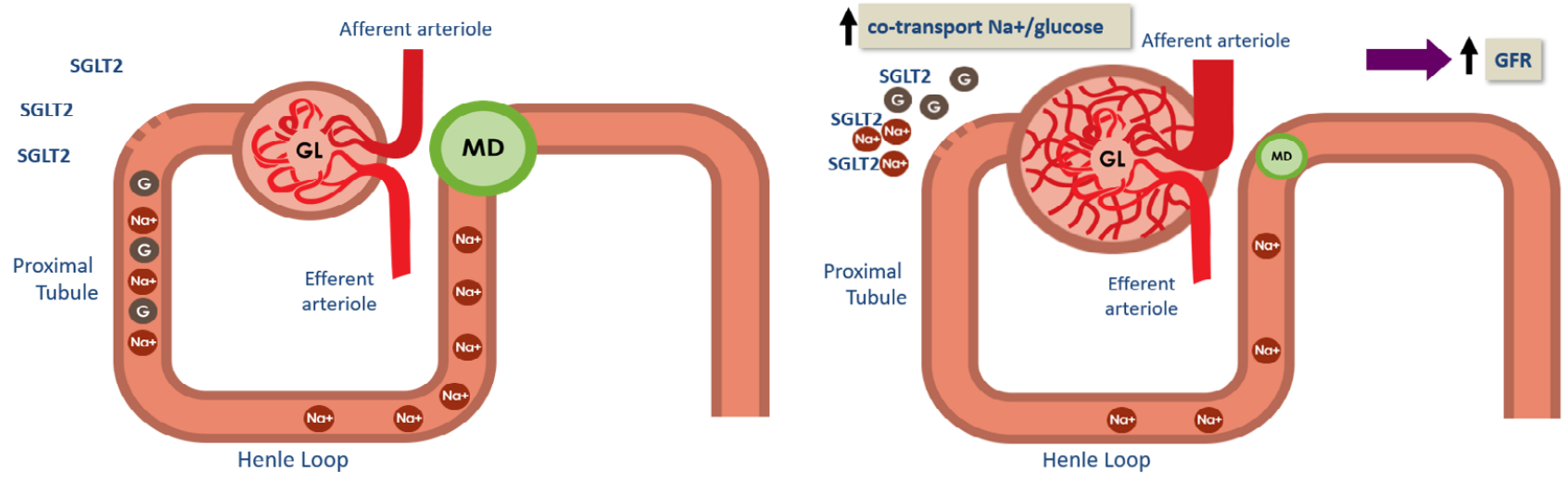
back, resembling a situation of renal hypoperfusion, the renin-angiotensin-aldosterone system (RAAS) is activated with efferent arteriolar vasoconstriction and afferent arteriolar vasodilation, generating increased intraglomerular and GFR pressure; over time, this hemodynamic mechanism results in injury of the glomeruli' ${ }^{12}$ (Figure 1). The consequences of diabetes mellitus, such as exacerbated production of growth factors and the aforementioned changes in glomerular hemodynamics, lead to hyperfiltration and glomerular hypertension, renal hypertrophy, and changes in the glomerular structure, with clinical manifestation typical of kidney disease caused by diabetes: albuminuria and hypertension.

\section{SGLT2 INHIBITION AS A THERAPEUTIC TARGET IN DIABETES MELLITUS AND IN NEPHROPROTECTION}

Although this is a current issue that has been frequently discussed in recent years, the renal SGLT inhibition was originated in the 1980s, still in experimental studies. On that occasion, it was found that the administration of Phlorizin, a non-selective SGLT inhibitor, reduced hyperglycemia in rats, in addition to improving insulin resistance; however, there was reduced bioavailability, in addition to gastrointestinal effects that hindered its application in clinical studies $^{13}$. This class of drugs has evolved and, currently, there are three main representatives of selective SGLT2 inhibitors: empagliflozin, dapagliflozin, and canagliflozin.

The glycosuria action of this class of medication is due to three mechanisms of action: the reduction of Tmg; reduction of glycosuria threshold; increase

FIGURE 2. GLOMERULAR HEMODYNAMIC EFFECT AS A RESULT OF SGLT2 INHIBITION.

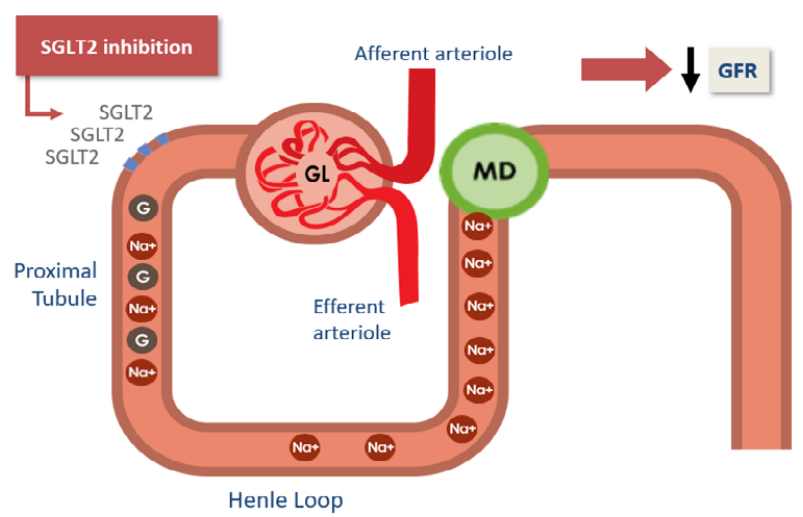

in the linear transition between reabsorption and renal excretion of glucose as the Tmg is reached ${ }^{9}$. When examined experimentally, the increase of glucose in urine occurred both in diabetic and normoglycemic patients, and the reduction of the glucose threshold appears to be the most significant pharmacological mechanism ${ }^{14}$.

The lower glucose reabsorption at the proximal tubule increases the availability of sodium and chloride that reaches the distal portions of the nephron, especially the macula densa. Thus, the greater intake of sodium chloride by this structure leads to the local release of adenosine and, consequently, of intracellular calcium, generating afferent arteriolar vasoconstriction ${ }^{15,16}$. This results in a reduction of glomerular hyperfiltration and, consequently, of intraglomerular hypertension, attenuating albuminuria, which are primary pathophysiological components in the development of kidney disease caused by diabetes, which explains the medication benefits beyond the blood glucose reduction ${ }^{17}$ (Figure 2).

In addition to the beneficial hemodynamic action, other effects also seem to contribute positively regarding the renal condition in diabetes mellitus; however, in a smaller proportion. The inhibition of SGLT2 reduces renal energy requirements, thus reducing renal hypoxia ${ }^{18}$. There is also an antioxidant, antifibrotic, and anti-inflammatory action due to the reduction of glycosylation products and the expression of inflammatory molecules ${ }^{19}$. Therefore, it is essential to stress the idea that the renal benefit from the SGLT2 action comes mostly from the glomerular hemodynamic effect to the detriment of the glucosuric effect alone, and, supposedly, hypoglycemic - which can vary from $0.4 \%$ to $1.1 \%$ of the glycated hemoglobin, in addition to reducing body weight ${ }^{20}$.

\section{RELEVANT CLINICAL STUDIES ON BENEFITS AND SECURITY}

To date, three large clinical trials have studied the cardiovascular and renal benefits of SGLT2 inhibitors, namely: EMPA-REG Outcome (empagliflozin), CANVAS (canagliflozin), and DECLARE-TIMI 58 (dapagliflozin) ${ }^{21-23}$. More recently, CREDENCE assessed in a primary way the renal outcomes with the use of canagliflozin ${ }^{24}$; we have detailed such evidence, and the data are summarized in Table 1.

EMPA-REG Outcome was a clinical trial, double-blind, that randomized 7,028 patients with type 
2 diabetes mellitus and established cardiovascular disease in 42 countries, who were divided into three groups: placebo, $10 \mathrm{mg}$, and $25 \mathrm{mg}$ of empagliflozin, with a three-year follow-up. The medication was added to the standard therapy (metformin and other hypoglycemic agents, as indicated by the clinical context of each patient). In the empagliflozin group, for both doses used, there was a significant reduction of cardiovascular mortality, a $32 \%$ reduction of general mortality, in addition to a $35 \%$ reduction of hospitalization due to heart failure. However, for acute myocardial infarction (AMI) and fatal and non-fatal stroke, there were no statistically significant differences $^{21}$. Subsequently, the population studied in the EMPA-REG Outcome was studied specifically for renal outcomes, i.e., progression to macroalbuminuria, doubling of serum creatinine, initiation of renal replacement therapy (RRT), and death due to kidney failure $^{25}$. It is worth noting that most individuals (approximately $75 \%$ ) had an estimated glomerular filtration rate (EGFR) above $60 \mathrm{ml} / \mathrm{min} / 1.73 \mathrm{~m}^{2}$ ), and $40 \%$ had an albuminuria/creatinine ratio (UACR) above $30 \mathrm{mg} / \mathrm{g}$ (population with incipient kidney disease, which limited more robust evaluations of the renal outcomes). Other than that, there were positive results in the aspects analyzed, and it was inferred that the patients in the group treated with empagliflozin had a reduction in the progression of the chronic kidney disease and lower incidence of clinically significant renal events. It was noted that, in both dose groups treated with the SGLT2 inhibitor, there was a decrease of up to $4 \mathrm{ml} / \mathrm{min} / 1.73 \mathrm{~m}^{2}$ in the first four weeks of treatment, which remained stable and without progression, unlike in the placebo group, in which there was a progressive decrease of eGFR during the period studied. This decrease in the first month of treatment is justified by the intraglomerular hemodynamic alteration, by the glomerular-tubule feedback mechanism with afferent arteriolar vasoconstriction, as previously explained. In regard to the safety of the treatment, the only significant finding was genital infection. The risk of worsening of renal function, dehydration, and fracture did not differ between the groups studied ${ }^{25}$.

The CANVAS, a randomized, multicenter clinical trial that included 10,142 patients in two study designs (Canvas and Canvas-R), had as its primary outcome to analyze cardiovascular mortality, non-fatal AMI, and non-fatal stroke. The trial included patients with type 2 diabetes mellitus and established cardio- vascular disease or with the presence of two risk factors for cardiovascular disease. In Canvas, there was randomization into three groups: canagliflozin $100 \mathrm{mg}$, canagliflozin $300 \mathrm{mg}$, and placebo, while in Canvas- $R$, there were two groups: canagliflozin 100 $\mathrm{mg}$ (with an optional increase to $300 \mathrm{mg}$ during follow-up) and placebo. Following the trends of analyzes for empagliflozin, the Canvas showed a reduction in cardiovascular mortality and hospitalization due to heart failure, with no significant impact on stroke and non-fatal AMI. When analyzed from the nephrological point of view, the average eGFR, from basic characteristics in all groups, was $76 \mathrm{ml} / \mathrm{min} / 1.73 \mathrm{~m}^{2}$, and $30 \%$ of individuals presented some degree of UACR $(7 \%$ above $300 \mathrm{mg} / \mathrm{g})$. In patients treated with the canagliflozin, there was a reduction in the progression of albuminuria, need for RRT, eGFR in 40\%, or renal death. The noteworthy significant adverse effects were an increase in the incidence of genital infections, greater osmotic diuresis, and reduced blood volume in the canaglifozin groups ${ }^{22}$. Other adverse effects, which previously had not been noticed, were a higher incidence of fractures and amputations in patients treated with the canagliflozin. Regarding the risk of amputation, no precise physiopathological explanation was found for it; it is possibly associated with episodes of dehydration and consequent hemoconcentration in individuals with previous peripheral arterial vascular involvement. A prior retrospective analysis also noted that risk as an effect of the SGLT2 inhibitor class, warning about the need for caution in susceptible patients, and monitoring for security in the long term ${ }^{26}$.

Another randomized, multicenter clinical trial, the DECLARE-TIMI 58, aimed to evaluate the clinical outcomes of dapagliflozin. In it, 17,160 patients with type 2 diabetes and established cardiovascular disease or high cardiovascular risk were studied by an average period of four years. Two groups were analyzed: a treatment group, which received $10 \mathrm{mg}$ of dapagliflozin, and a placebo group. Similar to previous trials, there was a benefit in the treatment group, with a reduction in cardiovascular mortality and hospitalization due to heart failure, but with no significance in the reduction of stroke and non-fatal AMI. There was also no reduction in the incidence of major cardiovascular effects, which is a possible explanation for the exclusion of patients with eGFR below $60 \mathrm{ml} / \mathrm{min} / 1.73 \mathrm{~m}^{2}$ (a group known to be of higher cardiovascular risk), which differs from pre- 


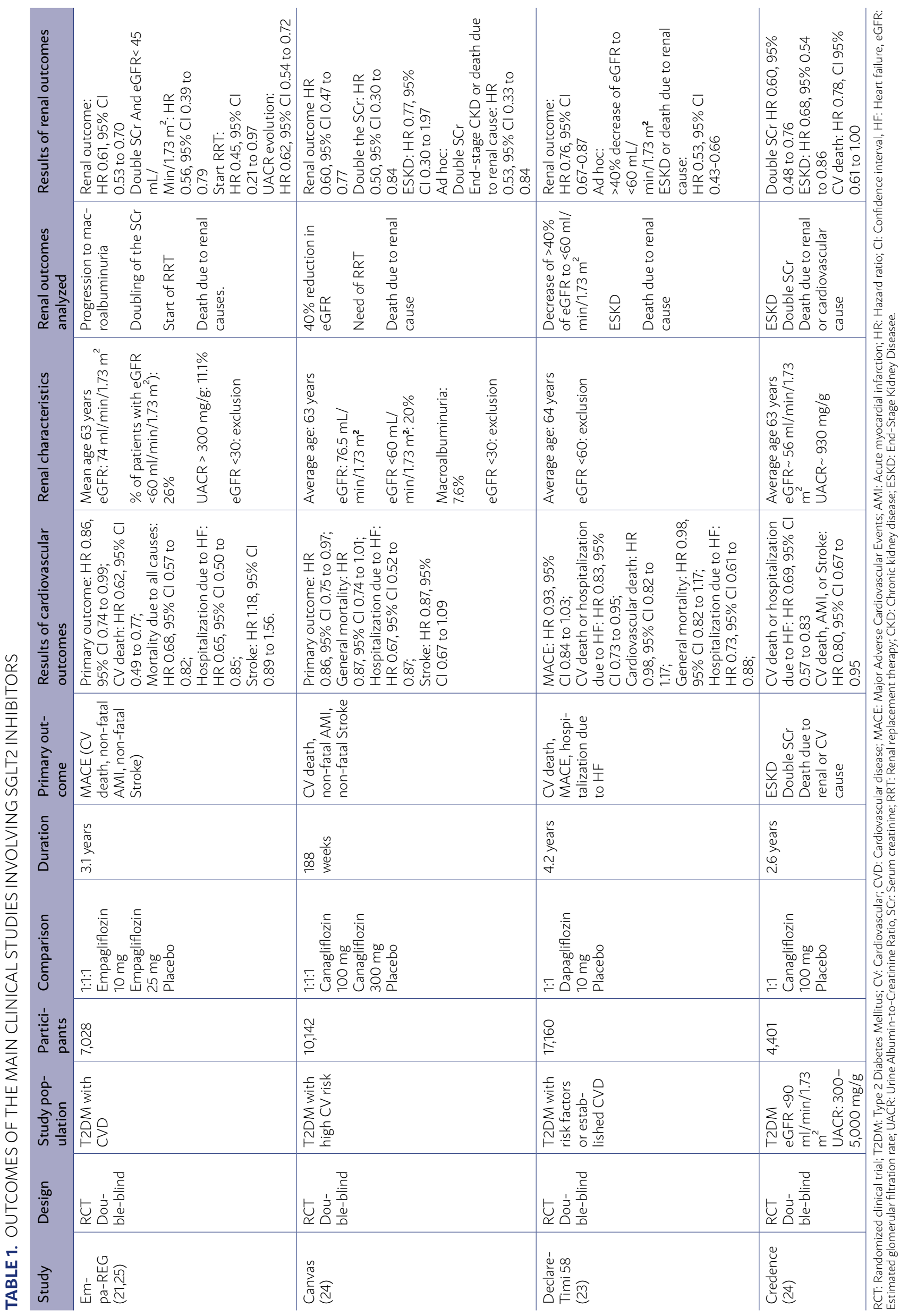


vious clinical studies. Despite this, when the renal outcomes were analyzed, there was a reduction in the incidence of end-stage kidney disease, a decrease greater than $40 \%$ in the eGFR, and death due to kidney failure. Basal levels and progression of albuminuria were not included in the analysis. Genital infection and diabetic ketoacidosis were adverse effects described as significant in the group treated with dapagliflozin ${ }^{23}$.

In these three large clinical trials, we note that, in relation to renal outcomes, individuals with no or little albuminuria were selected, as well as with normal or slightly reduced eGFR, which makes it difficult to make more complex analyses since this population has a low risk for progression of kidney disease. In this context, the CREDENCE study randomized 4,401 patients into a placebo group or a treatment group with $100 \mathrm{mg}$ of canagliflozin, with a follow-up of 2.6 years. Patients needed to meet the following inclusion criteria: type 2 diabetes with eGFR from 30 to $90 \mathrm{ml} /$ $\mathrm{min} / 1.73 \mathrm{~m}^{2}$, UACR of 300 to $5,000 \mathrm{mg} / \mathrm{g}$, and have an optimal and stable dose of angiotensin-converting enzyme inhibitors or angiotensin receptor blocker. Thus, the randomized groups included patients with an average of eGFR of $56 \mathrm{ml} / \mathrm{min} / 1.73 \mathrm{~m}^{2}$ and UACR of 930 $\mathrm{mg} / \mathrm{g}$. There was a reduction of $30 \%$ in the primary outcomes analyzed: end-stage kidney disease, doubling of serum creatinine, renal and cardiovascular mortality. For specific renal outcomes, the reduction was $34 \%$. In the analysis of subgroups, $59 \%$ of the patients presented eGFR between 60 and $30 \mathrm{ml} / \mathrm{min} / 1.73$ $\mathrm{m}^{2}$, a percentage that presented significant outcomes (the subgroup of 45 to $60 \mathrm{ml} / \mathrm{min} / 1.73 \mathrm{~m}^{2}$ had a $53 \%$ reduction in specific renal risk). It should be emphasized that such improvements were independent of the reduction in glycated hemoglobin. The subgroups with UACR greater than $1,000 \mathrm{mg} / \mathrm{g}$ and lower than $1,000 \mathrm{mg} / \mathrm{g}$ were also studied, with positive results in patients with a higher degree of proteinuria. In contrast with the CANVAS results, there was no difference in the incidence of amputations and fractures between the groups analyzed; there was an active investigation of signs of lesions on patient's feet during protocol visits and, in case of suspicion of worsening of the peripheral arterial disease, the treatment could be interrupted ${ }^{24}$.

In order to strengthen the evidence for the population with more advanced chronic kidney disease, the DAPA-CKD (Clinical trials: NCT03036150) is being conducted and will include patients with eGFR between 25 and $75 \mathrm{ml} / \mathrm{min} / 1.73 \mathrm{~m}^{2}$ or UACR between 200 and $5,000 \mathrm{mg} / \mathrm{g}$, with completion expected by November 2020 and the purpose of assessing the renal benefits of dapagliflozin in patients with chronic kidney disease, whether or not diabetic ${ }^{27}$. Another promising clinical trial is the Empa-Kidney (Clinical trials: NCT03594110), which will include patients with eGFR from 20 to $45 \mathrm{ml} / \mathrm{min} / 1.73 \mathrm{~m}^{2}$ and 45 to $90 \mathrm{ml} / \mathrm{min} / 1.73 \mathrm{~m}^{2}$ and UACR greater than $200 \mathrm{mg} / \mathrm{g}$; diabetic patients, or with established cardiovascular disease and eGFR $>60 \mathrm{ml} / \mathrm{min} / 1.73 \mathrm{~m}^{2}$ will be excluded. The primary goals are to evaluate the progression of kidney disease and cardiovascular death, and the study is expected to be completed by $2022^{28}$.

In smaller proportions, the SGLT2 inhibitors, in particular, empagliflozin, were also studied in another scenario of chronic kidney disease: post-transplant diabetes mellitus. In a small, non-randomized study, empagliflozin proved to be safe in these individuals, with a low risk of serious adverse effects. It had a low impact on the reduction of blood glucose levels, but significant weight reduction; there was not enough power to assess graft survival. This study emphasizes the safety of the medication and maintains the subject open to larger trials to expand results for the population of solid organ transplantation, in particular to kidney transplantation recipients ${ }^{29}$.

In relation to the safety profile of SGLT2 inhibitors, in general, they are well tolerated, safe medications, whose main significant adverse effect described were fungal genital infections (vulvovaginitis and balanoposthitis). Volume depletion, amputations, and fractures (these last two seen only with canagliflozin), although a controversial impact in trials, are described and deserve attention by the prescribing physician. Diabetic ketoacidosis, in particular, the euglycemic subtype, is classically associated with users of this medication who have type 1 diabetes mellitus - which actually hinders its use in this profile of patients. However, this was also observed in type 2 diabetic patients, so attention should be paid when the individual under treatment presents metabolic acidosis of unknown etiology ${ }^{30}$. Urinary tract infections were initially a cause for concern; however, subsequent strong analysis of the therapy did not correlate it with a higher incidence of urinary tract infections of any severity ${ }^{31}$. As more studies are awaited to better understand the extent of benefits, the safety assessment of 
SGLT2 inhibitors will continue to be discussed, especially in the population of greater risk, that is, that with impaired renal function. After consideration, without a doubt, the benefits of SGLT2 inhibition outweigh the risks; however, good clinical practice must be based on patient safety first, and the monitoring of adverse reactions should be thorough and continuous.

\section{PRACTICAL RECOMMENDATIONS FOR THE USE OF THE SGLT2 INHIBITORS IN CLINICAL PRACTICE}

The recommendation for initial treatment, according to the American Diabetes Association (ADA), is still metformin combined with changes in lifestyle. The cost-benefit relationship of the medication, allied to cardiovascular and metabolic benefits, justifies this as the first choice in the therapeutic $\operatorname{line}^{32}$. As adjuvant pharmacological therapy, the ADA recommends associating, in individuals with established cardiovascular disease, a SGLT2 inhibitor or glucagon-like peptide 1 (GLP-1) agonist. The GLP-1 agonists, represented mainly by exenatide, liraglutide, and semaglutide, belong to the group of incretin mimetics, intestinal hormones which potentiate the secretion of insulin, optimizing blood glucose levels, in addition to having cardio and renoprotective pleiotropic effects, although its clinical outcomes are less impactful ${ }^{33}$. In patients with risk of or established heart failure, there is a preference for the SGLT2 inhibitor, in view of the evidence above. If poor glycemic values persist, it is recommended that another hypoglycemic medication is added ${ }^{34}$. One alternative is the class of dipeptidyl peptidase 4 (DPP4) inhibitors, which have a different mechanism of action than the glycosuric drugs; the inhibition of this enzyme leads to the stabilization of GLP1, which justifies the glu- cose reduction effect. The impacts on cardiovascular and renal outcomes were controversial. However, recently, benefits in the short-term improvement of glycated hemoglobin and albuminuria were observed when used concomitantly to SGLT2 inhibitors, with good tolerability and security profile ${ }^{35}$.

Regarding the use of SGLT2 inhibitors in patients with reduced renal function, the recommendation is to use empagliflozin and canagliflozin when the eGFR is greater than $30 \mathrm{ml} / \mathrm{min} / 1.73 \mathrm{~m}^{2}$; dapagliflozin should be used when the eGFR is of at least $60 \mathrm{ml} /$ $\min / 1.73 \mathrm{~m}^{2}$. For the subpopulation with advanced CKD, when the other studies are completed, we will have answers regarding their efficacy and safety.

\section{CONCLUSION}

SGLT2 inhibitors are likely the greatest pharmacological advancement in nephrology since RAAS blockers; they are agents capable of reducing renal cardiovascular, a great foundation for oral hypoglycemic therapy ${ }^{36}$. With well-established clinical outcomes, they are already part of the therapeutic armamentarium against type 2 diabetes mellitus. Although limited for use in the Brazilian population, primarily due to its cost, its prescription should be instigated, and the cost-benefit relationship presented to the patient. Future publications are promising for the non-diabetic population, with even greater hopes of renal survival in patients with CKD.

\section{Contribution of the authors}

Diego Ennes Gonzalez, Renato Demarchi Foresto, and Artur Beltrame Ribeiro contributed substantially to the planning, drafting, and revision of this paper.

There is no conflict of interest in the present study.

There was no external funding.

\section{REFERENCES}

1. WHO. Global report on diabetes 2016. [cited 2019 Aug 12]. Available from: http://www.who.int/diabetes/global-report/en/

2. Eckardt KU, Coresh J, Devuyst O, Johnson RJ, Köttgen A, Levey AS, et al. Evolving importance of kidney disease: from subspecialty to global health burden. Lancet. 2013;382(9887):158-69.
3. Go AS, Chertow GM, Fan D, McCulloch CE, Hsu CY. Chronic kidney disease and the risks of death, cardiovascular events, and hospitalization. N Engl J Med. 2004;351(13):1296-305.

4. Afkarian M, Sachs MC, Kestenbaum B, Hirsch IB, Tuttle KR, Himmelfarb J, et al. Kidney disease and increased mortality risk in type 2 diabetes. J Am Soc Nephrol. 2013;24(2):302-8. 
5. Sesso RC, Lopes AA, Thomé FS, Lugon JR, Martins CT. Brazilian Chronic Dialysis Survey 2016. Braz J Nephrol. 2017;39(3):261-6.

6. van Bommel EJ, Muskiet MH, Tonneijck L, Kramer MH, Nieuwdorp M, van Raalte DH. SGLT2 inhibition in the diabetic kidney-from mechanisms to clinical outcome. Clin J Am Soc Nephrol. 2017;12(4):700-10.

7. Wright EM, Loo DD, Hirayama BA. Biology of human sodium glucose transporters. Physiol Rev. 2011;91(2):733-94.

8. Abdul-Ghani MA, Norton L, DeFronzo RA. Role of sodium-glucose cotransporter 2 (SGLT2) inhibitors in the treatment of type 2 diabetes. Endocr Rev. 2011;32(4):515-31.

9. DeFronzo RA, Norton L, Abdul-Ghani M. Renal, metabolic and cardiovascular considerations of SGLT2 inhibition. Nat Rev Nephrol. 2016;13(1):11-26.

10. Umanath K, Lewis JB. Update on diabetic nephropathy: core curriculum 2018. Am J Kidney Dis. 2018;71(6):884-95.

11. Anders HI, Huber TB, Isermann B, Schiffer M. CKD in diabetes: diabetic kidney disease versus nondiabetic kidney disease. Nat Rev Nephrol. 2018;14(6):361-77.

12. Tonneijck L, Muskiet MH, Smits MM, van Bommel EJ, Heerspink HJ, van Raalte DH, et al. Glomerular hyperfiltration in diabetes: mechanisms, clinical significance, and treatment. J Am Soc Nephrol. 2017;28(4):1023-39.

13. Rossetti L, Smith D, Shulman Gl, Papachristou D, DeFronzo RA. Correction of hyperglycemia with phlorizin normalizes tissue sensitivity to insulin in diabetic rats. J Clin Invest. 1987;79(5):1510-5.

14. DeFronzo RA, Hompesch M, Kasichayanula S, Liu X, Hong Y, Pfister M, et al. Characterization of renal glucosereabsorption in response to dapagliflozin in healthy subjects and subjects with type 2 diabetes. Diabetes Care. 2013;36(10):3169-76

15. Cherney DZ, Perkins BA, Soleymanlou N, Maione M, Lai V, Lee A, et al. Renal hemodynamic effect of sodium-glucose cotransporter 2 inhibition in patients with type 1 diabetes mellitus. Circulation. 2014;129(5):587-97.

16. Heerspink HJ, Perkins BA, Fitchett DH, Husain M, Cherney DZ. Sodium glucose cotransporter 2 inhibitors in the treatment of diabetes mellitus: cardiovascular and kidney effects, potential mechanisms, and clinical applications. Circulation. 2016;134(10):752-72.

17. Škrtić M, Cherney DZI. Sodium-glucose cotransporter-2 inhibition and the potential for renal protection in diabetic nephropathy. Curr Opin Nephrol Hypertens. 2015;24(1):96-103.

18. O'Neill J, Fasching A, Pihl L, Patinha D, Franzén S, Palm F. Acute SGLT inhibition normalizes $\mathrm{O} 2$ tension in the renal cortex but causes hypoxia in the renal medulla in anaesthetized control and diabetic rats. Am J Physiol Renal Physiol. 2015;309(3):F227-34

19. Ojima A, Matsui T, Nishino Y, Nakamura N, Yamagishi S. Empagliflozin, an inhibitor of sodium-glucose cotransporter 2 exerts anti-inflammatory and antifibrotic effects on experimental diabetic nephropathy partly by suppressing AGEs-receptor axis. Horm Metab Res. 2015;47(09):686-92.

20. Rosenstock J, Jelaska A, Frappin G, Salsali A, Kim G, Woerle HJ, et al. Improved glucose control with weight loss, lower insulin doses, and no increased hypoglycemia with empagliflozin added to titrated multiple daily injections of insulin in obese inadequately controlled type 2 diabetes. Diabetes Care. 2014;37(7):1815-23.

21. Zinman B, Wanner C, Lachin JM, Fitchett D, Bluhmki E, Hantel S, et al EMPA-REG OUTCOME Investigators. Empagliflozin, cardiovascular outcomes, and mortality in type 2 diabetes. N Engl J Med. 2015;373(22):2117-28.
22. Neal B, Perkovic V, Mahaffey KW, Zeeuw D, Fulcher G, Erondu N, et al. Canagliflozin and cardiovascular and renal events in type 2 diabetes. $N$ Engl Med. 2017;377(7):644-57.

23. Wiviott SD, Raz I, Bonaca MP, Mosenzon O, Kato ET, Cahn A, et al; DECLARE-TIMI 58 Investigators. Dapagliflozin and cardiovascular outcomes in type 2 diabetes. N Engl J Med. 2018;380(4):347-57.

24. Perkovic V, Jardine MJ, Neal B, Bompoint S, Heerspink HJL, Charytan DM, et al. Canagliflozin and renal outcomes in type 2 diabetes and nephropathy. N Engl I Med. 2019;380(24):2295-306.

25. Wanner C, Inzucchi SE, Lachin JM, Fitchett D, von Eynatten M, Mattheus M, et al; EMPA-REG OUTCOME Investigators. Empagliflozin and progression of kidney disease in type 2 diabetes. N Engl J Med. 2016;375(4):323-34.

26. Chang HY, Singh S, Mansour O, Baksh S, Alexander GC. Association between sodium-glucose cotransporter 2 inhibitors and lower extremity amputation among patients with type 2 diabetes. association between sodium-glucose cotransporter 2 inhibitors and lower extremity amputation among patients with type 2 diabetes. JAMA Intern Med. 2018;178(9):1190-8.

27. A study to evaluate the effect of dapagliflozin on renal outcomes and cardiovascular mortality in patients with chronic kidney disease (Dapa-CKD). [cited 2019 July 19]. Available from: https://clinicaltrials.gov/ct2/show/ results/NCT03036150

28. A Multicentre International Randomized Parallel Group Double-blind Placebo-controlled Clinical Trial of EMPAgliflozin Once Daily to Assess Cardio-renal Outcomes in Patients With Chronic KIDNEY Disease. [cited 2019 July 19]. Available from: https://clinicaltrials.gov/ct2/show/NCT03594110

29. Schwaiger E, Burghart L, Signorini L, Ristl R, Kopecky C, Tura A, et al. Empagliflozin in posttransplantation diabetes mellitus: a prospective, interventional pilot study on glucose metabolism, fluid volume, and patient safety. Am J Transplant. 2019;19(3):907-19.

30. Lupsa BC, Inzucchi SE. Use of SGLT2 inhibitors in type 2 diabetes: weighing the risks and benefits. Diabetologia. 2018;61(10):2118-25.

31. Dave CV, Schneeweiss S, Kim D, Fralick M, Tong A, Patorno E. Sodium-glucose cotransporter-2 inhibitors and the risk for severe urinary tract infections: a population-based cohort study. Ann Intern Med. 2019. doi: 10.7326/ M18-3136.

32. UK Prospective Diabetes Study (UKPDS) Group. Effect of intensive blood-glucose control with metformin on complications in overweight patients with type 2 diabetes (UKPDS 34). Lancet. 1998;352(9131):854-65.

33. Muskiet MHA, Tonneijck L, Smits MM, van Baar M|B, Kramer MHH, Hoorn EJ, et al. GLP-1 and the kidney: from physiology to pharmacology and outcomes in diabetes. Nat Rev Nephrol. 2017;13(10):605-28.

34. American Diabetes Association. 9. Pharmacologic approaches to glycemic treatment: standards of medical care in diabetes-2019. Diabetes Care. 2019;42(Suppl 1):S90-S102.

35. Pollock C, Stefánsson B, Reyner D, Rossing P, Sjöström CD Wheeler DC, et al. Albuminuria-lowering effect of dapagliflozin alone and in combination with saxagliptin and effect of dapagliflozin and saxagliptin on glycaemic control in patients with type 2 diabetes and chronic kidney disease (DELIGHT): a randomised, double-blind, placebo-controlled trial. Lancet Diabetes Endocrinol. 2019;7(6):429-41.

36. Bakris GL. Major advancements in slowing diabetic kidney disease progression: focus on SGLT2 inhibitors. Am | Kidney Dis. 2019. doi: 10.1053/j. ajkd.2019.05.009 